\title{
Generalized quark number susceptibilities from fugacity expansion at finite chemical potential for $\mathrm{N}_{\mathrm{f}}=2$ Wilson fermions
}

\author{
Christof Gattringer, Hans-Peter Schadler \\ Institute of Physics, University of Graz, 8010 Graz, Austria
}

(Dated: June 9, 2021)

\begin{abstract}
Generalized susceptibilities of the net quark number have been proposed to be good probes for the transitions in the QCD phase diagram and for the search of a possible critical end point. In this article we explore a new strategy for computing quark number susceptibilities from lattice QCD via an expansion in the fugacity parameter $e^{\mu \beta}$. All quark number related bulk observables are particularly easy to access in this approach and we present results for generalized quark number susceptibilities up to 4 -th order. Ratios of these quantities are studied and compared with model calculations for the high- and low temperature regions up to a chemical potential of $\mu \beta \approx 1.0$.
\end{abstract}

PACS numbers: 11.15.Ha, 12.38.Gc

\section{INTRODUCTION}

In recent years heavy ion collision experiments started to directly probe the phase diagram of QCD. In particular results from RHIC and LHC shed some light on properties of the quark gluon plasma and the position of the crossover line. To fully explore the phase diagram theoretically we need to calculate thermodynamical quantities not only at finite temperature but also at finite baryon chemical potential. For finite temperature we can use lattice QCD, the only ab-initio method available. However, for finite baryon density lattice QCD faces the so-called complex action problem" (or "sign problem"). In that case the Boltzmann factor becomes complex and cannot be interpreted as a probability for importance sampling and the usual Monte Carlo approach fails. To learn something about the phase diagram at least for small chemical potential, different methods have been developed and over the last years results have been obtained using reweighting methods, complex Langevin techniques as well as Taylor expansion in the chemical potential $\mu$ (see, e.g., [1] for recent reviews).

In this paper we focus on a different expansion technique, the fugacity expansion. In this approach one expands the partition sum in a Laurent series in the fugacity parameter $z=e^{\mu \beta}$, where $\beta$ is the inverse temperature and $\mu$ is the chemical potential. This expansion is a rather natural choice, since the chemical potential on the lattice may be introduced as a boundary term by multiplying the factor $e^{\mu \beta}\left(e^{-\mu \beta}\right)$ on the forward (backward) temporal hopping term of the fermion action that connects the last with the first timeslice. Furthermore, on a finite lattice the fugacity series is finite, while the Taylor expansion always gives rise to an infinite series.

For a first implementation of the fugacity expansion in QCD based on the strategy used here, see [2]. Preliminary results from this study and a variant for staggered fermions are documented in [3].

Recent comparisons of different expansion techniques to results from a dual variables simulation for a QCD related model have shown that the fugacity expansion may provide better convergence properties than the usual Taylor expansion [4]. Here we want to explore the possibilities of the fugacity expansion in full QCD by calculating bulk observables related to the quark number which are particularly simple in the fugacity expansion. In particular the quark number density and also higher generalized susceptibilities are evaluated and are compared to hadron resonance gas model calculations. The results we present are for ensembles of $N_{f}=2$ Wilson fermions on $N_{s}^{3} \times N_{t}=8^{3} \times 4$ and $12^{3} \times 6$ lattices for temperatures below and above the crossover.

\section{FUGACITY EXPANSION FOR WILSON FERMIONS}

The fugacity expansion is introduced in the following way: The partition function for $N_{f}=2$ mass degenerate quark flavors has the general form

$$
Z_{\mu}=\int D[U] e^{-S_{g}(U)} \operatorname{det}[D(\mu, U)]^{2},
$$

where $S_{g}(U)$ denotes the usual Wilson plaquette action and $D(\mu, U)$ is the Wilson Dirac operator with chemical potential $\mu$ in a background gauge configuration $U$ (from now on we omit the gauge field dependence as argument, but when useful display the dependence on the chemical potential $\mu$ ). The fermion determinant $\operatorname{det}[D(\mu)]$ can be written as a Laurent series in the fugacity parameter $z=e^{\mu \beta}$,

$$
\operatorname{det}[D(\mu)]=\sum_{q=-q_{\max }}^{q_{\max }} e^{\mu \beta q} D^{(q)},
$$

where $\beta=1 / T=N_{t}$ (the Boltzmann constant and the lattice spacing are set to 1 ). As mentioned in the introduction, the Taylor expansion is an infinite series also on 
a finite lattice. In contrast, the Laurent series of the fugacity expansion is finite, summing over all possible net quark numbers $q$ between $q=-q_{\max }$ and $q=+q_{\max }$ with $q_{\max }=2 \times 3 \times N_{s}^{3}$. Below we will show that it is justified to truncate the fugacity series at values of $q$ which are much smaller than $q_{\max }$.

The expansion coefficients $D^{(q)}$ are referred to as "canonical determinants" and can be computed as the Fourier moments with respect to imaginary chemical potential (compare also $[5,6]$ ),

$$
D^{(q)}=\frac{1}{2 \pi} \int_{-\pi}^{\pi} d \phi e^{-i q \phi} \operatorname{det}[D(\mu \beta=i \phi)] .
$$

They have the interpretation of projections of the grand canonical fermion determinant $\operatorname{det}[D(\mu)]$ to a fixed net quark number $q$, and also provide the basis for the canonical approach (see, e.g., [7]).

For large values of the net quark number the evaluation of the integral in (3) becomes numerically harder (see below) and in actual calculations $q$ is limited by $|q| \leq q_{\text {cut }}$, where $q_{\text {cut }}$ is much smaller than $q_{\max }$. Usually we can reach values up to $q_{\text {cut }}=60$ in the numerical evaluation depending on the coupling and the volume of the considered ensemble.

Equations (2) and (3) provide an insight on how the chemical potential works physically, which in turn leads to an understanding of the numerical challenges of the fugacity expansion: The key issue is the size distribution of the terms in the sum (2). In Fig. 1 we plot as a function of $q$ the expectation value of the absolute value of the summands normalized by $\left|D^{(0)}\right|$, i.e., we plot $\left\langle e^{\mu \beta q}\left|D^{(q)}\right| /\left|D^{(0)}\right|\right\rangle$ (for information about the complex phase of the $D^{(q)}$ see, e.g., [2]).

The $\mu=0$ data show the distribution of the $\left|D^{(q)}\right|$ without the fugacity factors. In the plot with the linear scale (top plot in Fig. 1) they display a Gaussianlike distribution centered around $q=0$. In addition to the linear scale, in the bottom plot we also show the $\left\langle e^{\mu \beta q}\left|D^{(q)}\right| /\left|D^{(0)}\right|\right\rangle$ using a log scale to better resolve the behavior in the tails of the distribution. When turning on the chemical potential the factors $e^{\mu \beta q}$ break the symmetry around $q=0$ and shift the distribution of the summands towards larger values of $q$. This is exactly the behavior that we expect from a chemical potential: The system exhibits an average net quark number which is different from zero. Note that the distribution for $\mu>0$ is the result of multiplying the factor $e^{\mu \beta q}$, which exponentially increases with $q$, with the Gaussian-type of decay of the $D^{(q)}$. This implies that the $D^{(q)}$ at large $q$ have to be evaluated very accurately to capture the necessary compensation of the exponential rise from the $e^{\mu \beta q}$. This necessary numerical accuracy is the limiting factor for the values of $\mu \beta$ one can reliably reach in the fugacity expansion.

Let us now come to the question of how to express observables related to the quark number in the fugacity series, i.e., in terms of the expansion coefficients $D^{(q)}$.
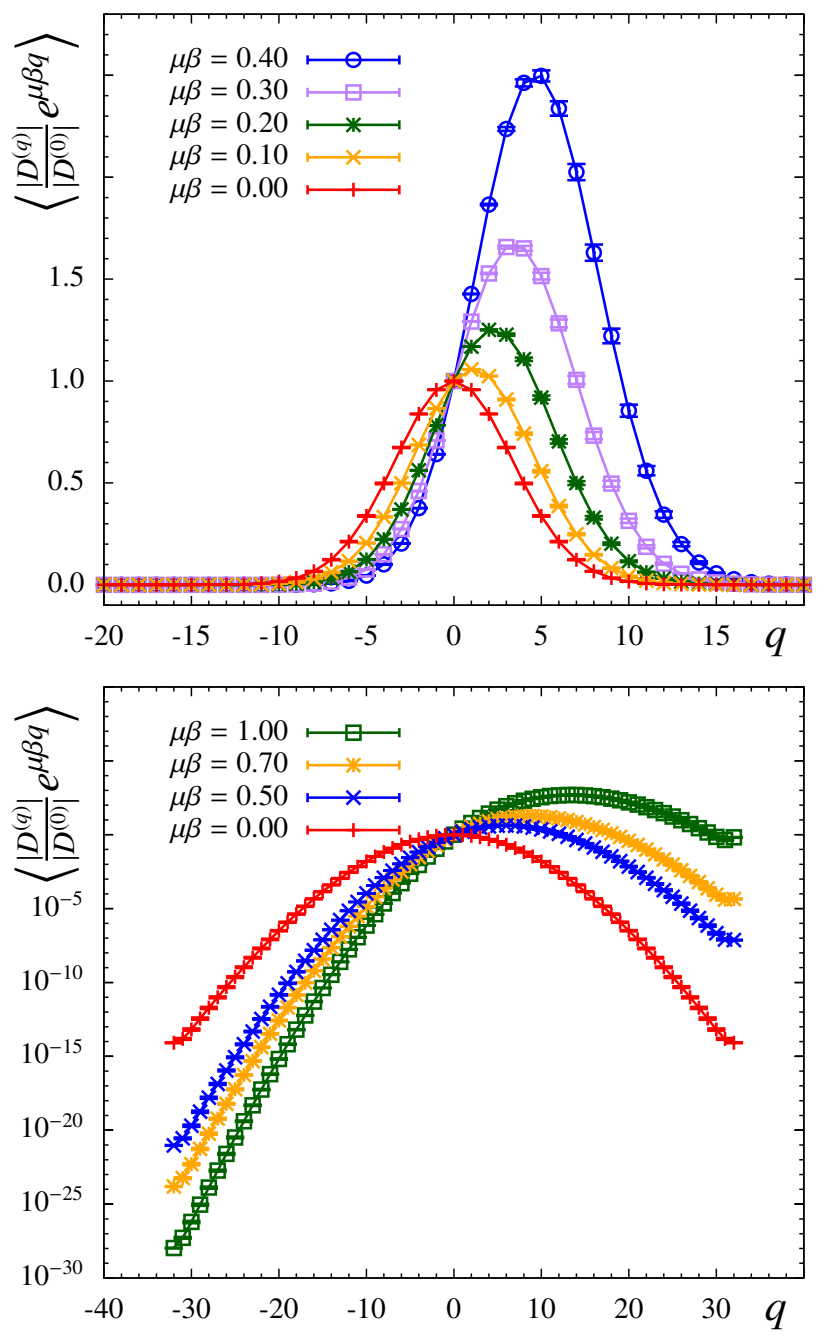

Figure 1: Distribution of the absolute values of the canonical determinants normalized by $\left|D^{(0)}\right|$ and weighted with the fugacity factors as a function of the net quark number $q$. The results are from our $12^{3} \times 6, \kappa=0.162,6 / g^{2}=5.40$ ensemble. In the top plot we use a linear scale and values of $\mu \beta$ up to 0.4 , while in the bottom plot a log-scale is used for values of $\mu \beta$ up to 1.0 .

We start with the partition function where we now express the determinant by the fugacity series from Eq. (2),

$$
\begin{aligned}
& Z_{\mu}= \int D[U] e^{-S_{g}[U]}\left(\sum_{q} e^{\mu \beta q} D^{(q)}\right)^{2} \\
&= \int D[U] e^{-S_{g}[U]} \operatorname{det}[D(\mu=0)]^{2} \times \\
& \times\left(\sum_{q} e^{\mu \beta q} \frac{D^{(q)}}{\operatorname{det}[D(\mu=0)]}\right)^{2} \\
&=\left\langle\left(M^{(0)}\right)^{2}\right\rangle_{0} Z_{0} .
\end{aligned}
$$

In the last step we have introduced the moments of the 
canonical determinants

$$
M^{(n)}=\sum_{q} e^{\mu \beta q} q^{n} \frac{D^{(q)}}{\operatorname{det}[D(\mu=0)]} .
$$

The expectation values $\langle\ldots\rangle_{0}$ are evaluated on configurations generated with vanishing chemical potential $\mu=0$. This implies that the fugacity series has the overlap problem in the same way as all expansion approaches, in particular also the Taylor series.

In this study we consider bulk observables related to the quark number which are given by derivatives of the partition function with respect to the chemical potential $\mu$, i.e., the generalized susceptibilities $\left(V=N_{s}^{3}\right)$ :

$$
\chi_{n}^{q}=\frac{1}{V \beta} \frac{\partial^{n} \ln Z_{\mu}}{\partial \mu^{n}} .
$$

Here the label $q$ on the lhs. is used to distinguish the generalized quark number susceptibilities from other commonly used susceptibilities in QCD. Using Eq. (4) the first derivative, i.e., the quark number density, can be easily calculated and is given by

$$
\frac{\chi_{1}^{q}}{T^{3}}=\frac{n_{q}}{T^{3}}=2 \frac{\beta^{3}}{V} \frac{\left\langle M^{(0)} M^{(1)}\right\rangle_{0}}{\left\langle\left(M^{(0)}\right)^{2}\right\rangle_{0}} .
$$

The result is expressed in terms of the moments defined in Eq. (5). Another derivative gives the quark number susceptibility

$$
\begin{aligned}
\frac{\chi_{2}^{q}}{T^{2}}=2 \frac{\beta^{3}}{V} & {\left[\frac{\left\langle\left(M^{(1)}\right)^{2}\right\rangle_{0}+\left\langle M^{(0)} M^{(2)}\right\rangle_{0}}{\left\langle\left(M^{(0)}\right)^{2}\right\rangle_{0}}\right.} \\
& \left.-2\left(\frac{\left\langle M^{(0)} M^{(1)}\right\rangle_{0}}{\left\langle\left(M^{(0)}\right)^{2}\right\rangle_{0}}\right)^{2}\right] .
\end{aligned}
$$

It is straightforward to express further derivatives via the moments $M^{(n)}$, and for $\chi_{3}^{q}$ and $\chi_{4}^{q}$, which we also consider here, we do not display the simple but somewhat lengthy expressions. $\chi_{3}^{q}$ and $\chi_{4}^{q}$ will be particularly useful when we study ratios of generalized susceptibilities which have nice properties, not only for numerical tests, but are also convenient for a comparison with experiment [8].

\section{EVALUATION AND PROPERTIES OF $\mathbf{D}^{(\mathrm{q})}$}

As we have already discussed, for accessing reasonably large values of $\mu \beta$ we need to very precisely calculate the canonical determinants $D^{(q)}$ up to a large net quark number $q$. Two factors crucially influence the accuracy when computing the $D^{(q)}$ using Eq. (3): The number of values of $\phi \in[-\pi, \pi]$ has to be sufficiently large when numerically computing the Fourier integrals and the integrand itself has to be evaluated at high precision.
For the results presented here we use 256 integration points for $\phi$, but we have also performed tests for larger and smaller numbers of points. In these tests we did not observe deviations of the results and we conclude that the integration is stable for the values of $q$ we take into account here.

For obtaining the necessary accuracy of the integrand in Eq. (3), i.e., for the precise calculation of the canonical determinant $\operatorname{det}[D(\mu \beta=i \phi)]$, we use an exact evaluation with LU factorization. This is of course a sizable numerical effort and to reduce the computing time and memory requirements we apply the dimensional reduction given in [9] (for a different dimensional reduction formula of the Wilson Dirac operator determinant see [10]). We here use a slightly modified form which is more suitable for our calculations (see the appendix for a detailed derivation). The dimensional reduction can be summarized as follows: The determinant of the Dirac operator can exactly be rewritten as

$$
\operatorname{det}[D(\mu)]=A W(\mu \beta),
$$

with

$$
W(\mu \beta)=\operatorname{det}\left[K_{0}-e^{\mu \beta} K-e^{-\mu \beta} K^{\dagger}\right],
$$

and a $\mu$-independent factor $A$ which cancels in the calculation of the observables. $K_{0}$ and $K$ are two dense matrices depending on the gauge fields, but not on the chemical potential $\mu$. We pre-compute them in our code, store them completely and then use them many times for evaluating $W(\mu \beta)$ with $\mu \beta=i \phi$ at all values $\phi$ needed for the numerical integration of Eq. (3). The dimension of the matrices $K_{0}$ and $K$ is $N_{s}^{3} \times 1 \times 4 \times 3$, in contrast to the original (sparse) Dirac operator which is $N_{s}^{3} \times N_{t} \times 4 \times 3$ dimensional. Since the cost of the exact evaluation of the determinant is of third order in the dimension of $D$, the exact dimensional reduction (9), (10) speeds up the evaluation by a factor proportional to $N_{t}^{3}$. With the computer resources available to us this procedure allows for a precise and cost efficient evaluation of $\operatorname{det}[D(\mu)]$ on lattices with sizes up to $12^{3} \times 6$.

The results we present in this article are for two different sets of dynamical Wilson fermion ensembles, $N_{s}^{3} \times N_{t}=8^{3} \times 4$ with an inverse mass parameter of $\kappa=0.158$ and $12^{3} \times 6$ with $\kappa=0.162$. The temperature is driven by varying the inverse coupling in the range of $6 / g^{2}=5.00$ up to $6 / g^{2}=5.70$. These parameters correspond to lattice spacings of approximately $a=0.320 \mathrm{fm}$ down to $a=0.150 \mathrm{fm}$ and to pion masses of $M_{\pi} \leq 960 \mathrm{MeV}$ (for $\kappa=0.158$ ) and $M_{\pi} \leq 900 \mathrm{MeV}$ (for $\kappa=0.162$ ), respectively. The errors are calculated using the Jackknife method with 300 configurations per parameter for the smaller lattices and 50 to 100 configurations for the larger lattices. To study volume effects we use data with different spatial volumes. All configurations were generated using the MILC collaboration public lattice gauge theory code [11].

Let us comment on the volume dependence of the numerical cost: The width of the distribution of the $D^{(q)}$ 


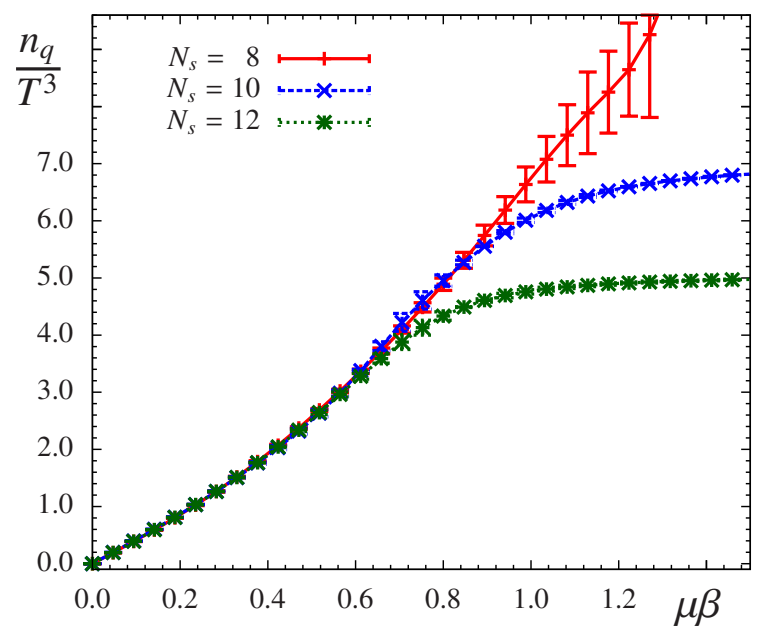

Figure 2: Comparison of the quark number density as a function of the chemical potential for three spatial volumes with $N_{s}=8, N_{s}=10$ and $N_{s}=12$. The results are for $N_{t}=4$, $\kappa=0.158$ and $6 / g^{2}=5.30$.

shown in Fig. 1 is related to the quark number susceptibility and thus is an extensive quantity, i.e., it grows with the 3 -volume $V$. Thus when increasing the volume, also $q_{c u t}$ has to grow accordingly and the $D^{(q)}$ have to be evaluated for larger values of $q$. In turn, when the number of $D^{(q)}$ that are used is kept fixed while increasing the volume, one expects that the fugacity series breaks down already at smaller values of $\mu \beta$.

This is illustrated in Fig. 2, where we study the quark number density as a function of $\mu \beta$ for three different spatial volumes at $\kappa=0.158$ and a coupling near the crossover, $6 / g^{2}=5.30$. The different spatial lattice extents are $N_{s}=8, N_{s}=10$ and $N_{s}=12$. Up to a value of $\mu \beta=0.6$ the results from the three volumes agree. At this point, however, the density of the largest volume becomes flat while for the other volumes it still rises. The statistical errors are still small, but nevertheless this signals the breakdown of the expansion at this point for the largest volume. When we increase the chemical potential to $\mu \beta=0.9$ also the $N_{s}=10$ result starts to deviate and becomes flat. The smallest lattice volume still shows the expected behavior, i.e., a rise with chemical potential. A similar behavior is seen in the volume dependence of the quark number susceptibility and we conclude that when increasing the volume, also $q_{c u t}$ has to be increased roughly linearly with $N_{s}^{3}$ (see also the discussion below).

As we have already mentioned above, the distribution of the canonical determinants $D^{(q)}$ governs the numerical cost of the fugacity expansion, and various ideas have been followed to describe the distribution $[12,13]$. In particular for the distribution of $\left|D^{(q)}\right|^{2}$ one expects a socalled Skellam distribution which, in a normalized form, is given by

$$
\frac{\left\langle\left|D^{(q)}\right|^{2}\right\rangle}{\left\langle\left|D^{(0)}\right|^{2}\right\rangle}=\frac{I_{q}(\zeta)}{I_{0}(\zeta)}
$$

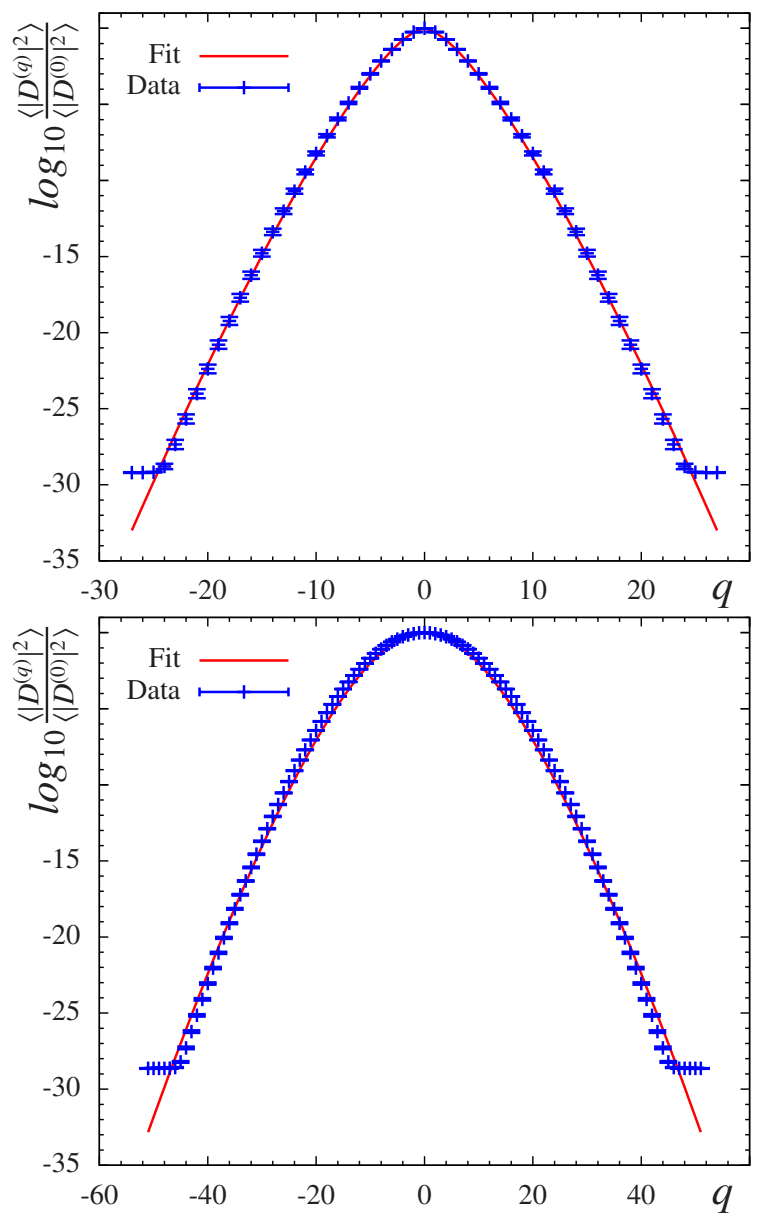

Figure 3: Logarithm of the normalized canonical determinants and a fit of the data to a Skellam distribution (Eq. (11)) are shown. The data is for the $8^{3} \times 4, \kappa=0.158$ ensemble at temperatures $T=144 \mathrm{MeV}$ (upper plot) and $T=211 \mathrm{MeV}$ (lower plot).

where the $I_{q}$ are modified Bessel functions. For a qualitative comparison, in Fig. 3 we show a fit of this distribution in the parameter $\zeta$ to data from our $8^{3} \times 4, \kappa=0.158$ ensemble. We use two temperatures, $T=144 \mathrm{MeV}$ below the crossover (upper plot) and $T=211 \mathrm{MeV}$ above the crossover (lower plot). Clearly visible is the strong dependence on the temperature: For low temperatures the width of the distribution is small compared to high temperatures where it is nearly twice as wide as in the $T=144 \mathrm{MeV}$ case. The flat areas for large values of $|q|$ are numerical artifacts and for the evaluation of the fugacity sum one has to stop at smaller $q$. Also for the fit to the Skellam distribution these points have been excluded.

From chiral perturbation theory one can derive formulas for various distributions of the canonical determinants, which make explicit also the dependence on the temperature and pion mass [13]. For the distribution considered in Eq. (11) it gives the explicit dependence of the argument $\zeta$ on the parameters $M_{\pi}, T$, as well as the spatial volume $V$. In particular $\zeta$ is proportional to the 

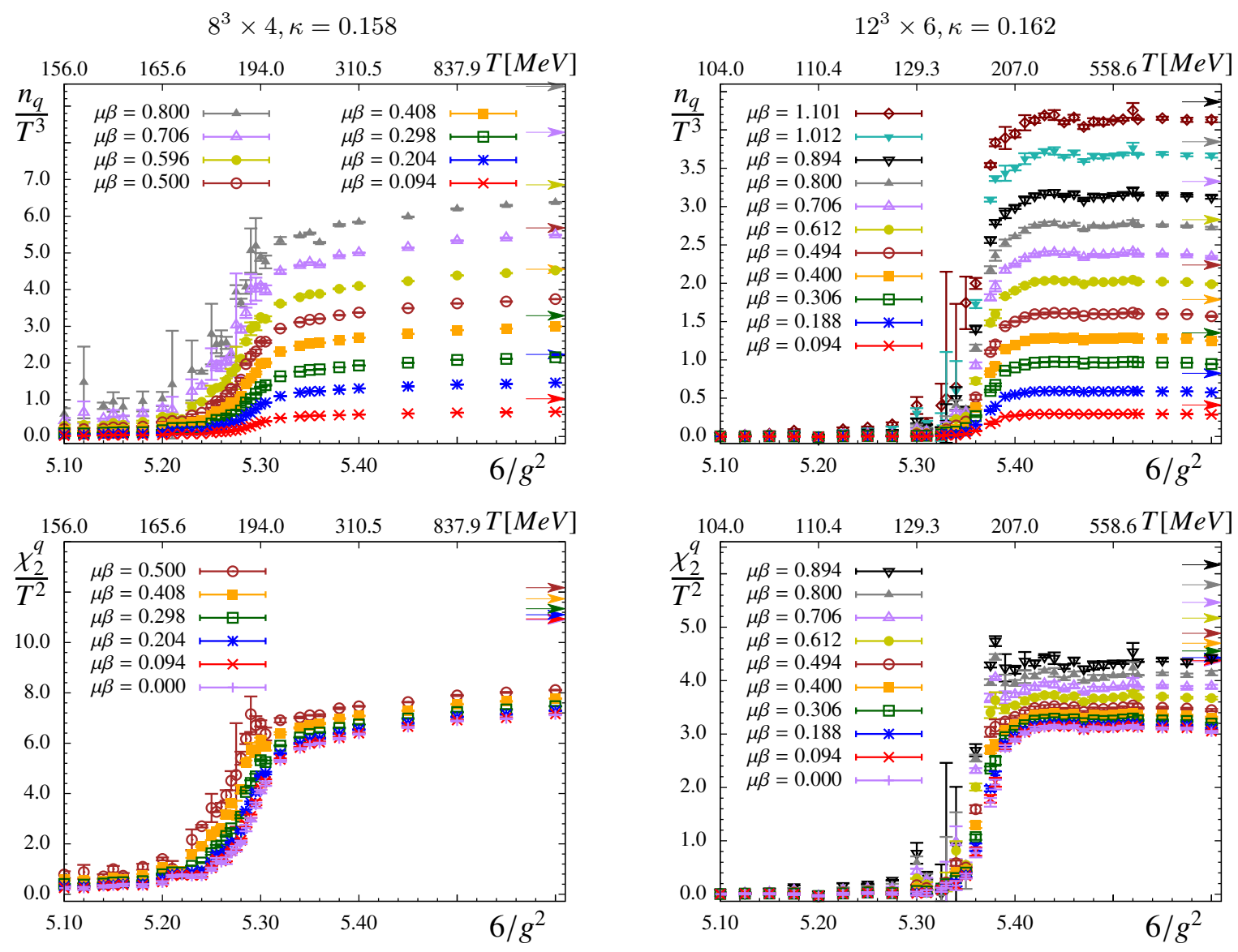

Figure 4: Quark number density $n_{q} / T^{3}$ and susceptibility $\chi_{2}^{q} / T^{2}$ as a function of the inverse coupling (corresponding temperature values on the top scale) for the $8^{3} \times 4, \kappa=0.158$ (lhs.) and $12^{3} \times 6, \kappa=0.162$ (rhs.) ensembles. The arrows on the rhs. of the plots mark the Stefan-Boltzmann high temperature limits.

spatial volume, implying that the width of the distribution increases roughly linearly with $V$, as stated above in the discussion of the numerical cost as a function of the volume. A detailed comparison of the Monte Carlo results to the distributions from chiral perturbation theory is presented in [13].

\section{HADRON RESONANCE GAS}

The hadron resonance gas (HRG) approach is based on the idea that in the confined region only quasi free hadrons appear. In this section we briefly collect and summarize the HRG formulas which we need for the comparison with our results. The basic ansatz of the HRG is to describe the system as a sum over free fermion partition functions for baryons and mesons (including resonances) with their appropriate masses [14]. Here we are only interested in the part which contributes to the quark number related observables, i.e., the baryonic part. This part can be written as

$$
\begin{aligned}
\ln Z_{B} & \approx \sum_{i} \frac{V d_{i} T}{\pi^{2}}\left[m_{i}^{2} K_{2}\left(m_{i} \beta\right)\right] \cosh (3 \mu \beta) \\
& =F(T, m) \cosh (3 \mu \beta) \approx \frac{p}{T^{4}} .
\end{aligned}
$$

Here $K_{2}$ denotes a modified Bessel function and the sum runs over all baryons with masses $m_{i}$. The introduction of the chemical potential gives rise to the cosh function, which we have separated in the third step from the mass dependent part denoted by $F(T, m)$.

It is obvious that ratios of derivatives with respect to $\mu$ are independent of this function $F(T, m)$ and therefore independent of the mass spectrum. Furthermore, the dependence on the chemical potential will either be via a $\tanh (3 \mu \beta)$ or $\operatorname{at} \operatorname{coth}(3 \mu \beta)$ or, for the case of even/even or odd/odd derivative ratios, independent of $\mu$. In particular we will use the following ratios

$$
\begin{aligned}
& \frac{n_{q} / T^{3}}{\chi_{2}^{q} / T^{2}}=\frac{1}{3} \tanh (3 \mu \beta), \\
& \frac{\chi_{3}^{q} / T}{\chi_{2}^{q} / T^{2}}=3 \operatorname{coth}(3 \mu \beta),
\end{aligned}
$$



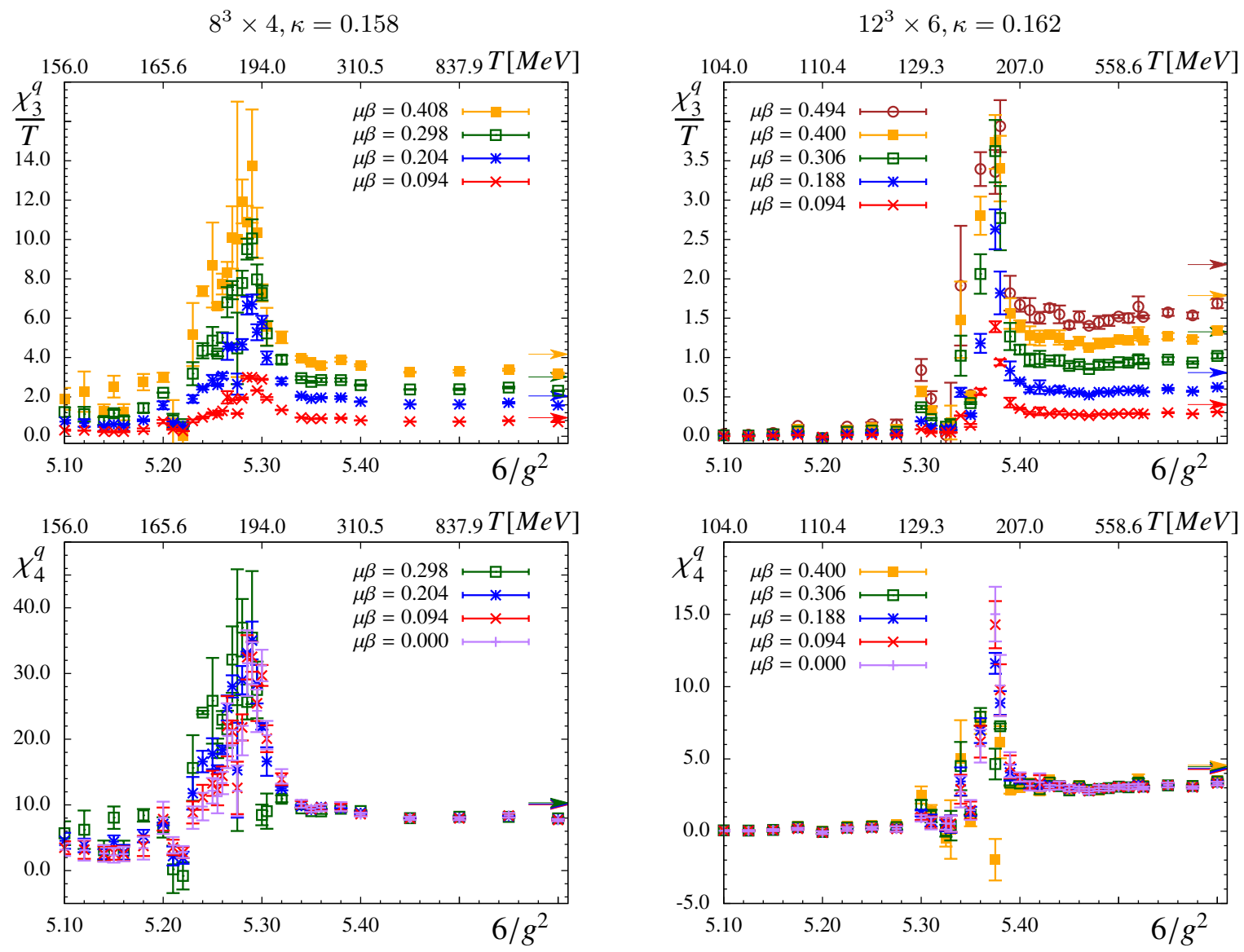

Figure 5: The generalized quark number susceptibilities $\chi_{3}^{q} / T$ (top) and $\chi_{4}$ (bottom) as a function of the inverse coupling (corresponding temperature values on the top scale) for the $8^{3} \times 4, \kappa=0.158$ (lhs.) and $12^{3} \times 6, \kappa=0.162$ (rhs.) ensembles. The arrows on the rhs. of the plots mark the Stefan-Boltzmann high temperature limits.

and

$$
\frac{\chi_{4}^{q}}{\chi_{2}^{q} / T^{2}}=9
$$

for comparison with the observables obtained using the fugacity expansion.

\section{NUMERICAL RESULTS}

We now come to the presentation of the results for the generalized susceptibilities and their ratios (for a strategy of comparing the lattice data to experiments see [15]). We compare the results for our two lattices, $8^{3} \times 4$ and $12^{3} \times 6$, with parameters as specified in Section III. Before showing the new results we remark that the $8^{3} \times 4$ data have parameters similar to the preliminary calculations with the fugacity expansion discussed in [2]. The new results agree with those from [2] within error bars, but they have smaller errors due to improvements in the numerics.

In Fig. 4 we now show the new results for the quark number density $n_{q} / T^{3}$ (upper plots) and the quark num- ber susceptibility $\chi_{2}^{q} / T^{2}$ (lower plots) as a function of the inverse coupling (corresponding temperature values on the top scale) for different values of the chemical potential $\mu \beta$. The plots on the lhs. are for $8^{3} \times 4, \kappa=0.158$ and $12^{3} \times 6, \kappa=0.162$ is used on the rhs.

For the quark number density $n_{q} / T^{3}$, we find a small but non-zero value below the crossover (for $\mu>0$; for $\mu=0$ one has $n_{q} \equiv 0$ for all temperatures). In the crossover region we observe a more rapid increase with $6 / g^{2}$ (respectively $T$ ) and above the crossover again a slow rising. Increasing the chemical potential $\mu$ essentially shifts the whole function for $n_{q} / T^{3}$ towards larger quark numbers. Qualitatively the behavior is the same for both lattice sizes. At very large temperatures one expects a free gas behavior (Stefan-Boltzmann behavior) and we mark the corresponding values by arrows on the rhs. of the plots. Obviously the temperatures we work at are not yet in the Stefan-Boltzmann region.

The second derivative with respect to the chemical potential, i.e., the quark number susceptibility, also shows the expected behavior: Below the crossover the curves are flat but non-zero while the crossover is marked by a sudden steep rise of $\chi_{2}^{q} / T^{2}$. Above the crossover 

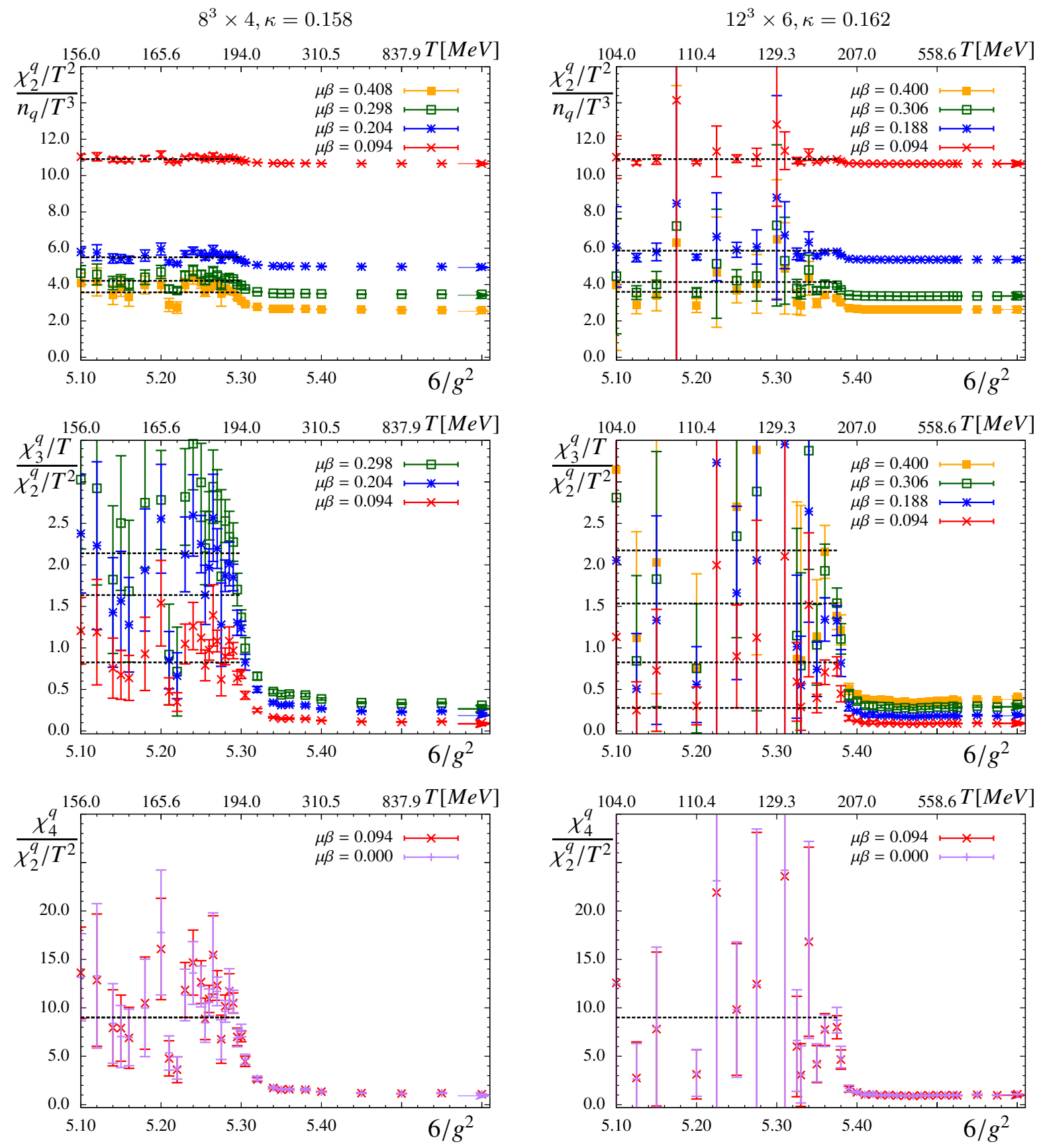

Figure 6: Ratios of the generalized quark number susceptibilities $\left(\chi_{2}^{q} / T^{2}\right) /\left(n_{q} / T^{3}\right),\left(\chi_{3}^{q} / T\right) /\left(\chi_{2}^{q} / T^{2}\right)$ and $\left(\chi_{4}^{q}\right) /\left(\chi_{2}^{q} / T^{2}\right)($ top to bottom) as a function of the inverse coupling (corresponding temperature values on the top scale) for the $8^{3} \times 4, \kappa=0.158$ (lhs.) and $12^{3} \times 6, \kappa=0.162$ (rhs.) ensembles. The dashed lines in the confined region are the HRG results and the arrows on the rhs. of the plots mark the Stefan-Boltzmann high temperature limits.

$\chi_{2}^{q} / T^{2}$ develops a plateau-like behavior with only a small slope, again undershooting the Stefan-Boltzmann values (marked by the arrows) and approaching them only slowly with temperature. It is obvious, that with increasing chemical potential the position of the steep rise of $\chi_{2}^{q} / T^{2}$ shifts towards smaller $T$. This corresponds to the bending of the crossover curve in the $\mu$-T diagram towards smaller temperatures when increasing $\mu$.
Our results for the higher derivatives $\chi_{3}^{q} / T$ and $\chi_{4}^{q}$ are shown in Fig. 5. Both observables peak in the crossover region near a pseudo-critical temperature. For $\chi_{3}^{q} / T$ and $\chi_{4}^{q}$ the error bars in the transition region are already quite sizable due to the large fluctuations at the crossover. This is also the reason, why less values of $\mu \beta$ could be realiably evaluated. For the values we show, the 4 th derivative displays only a rather small sensitivity to the chem- 
ical potential, which is visible mainly in the crossover region where unfortunately also the error bars are largest. For large temperatures both, $\chi_{3}^{q} / T$ and $\chi_{4}^{q}$ approach the Stefan-Boltzmann values, and we observe that the relative deviations of the higher derivatives are smaller than for $n_{q} / T^{3}$ and $\chi_{2}^{q} / T^{2}$.

From theoretical and experimental interest are ratios of the generalized susceptibilities. They are accessible in experiment and do not depend on the physical volume. In theory these quantities can give a clear signal of deconfinement with distinct behavior above, below and at the crossover. In addition they are essentially constant in the regions above and below the transition and one can compare them with model calculations like the hadron resonance gas (HRG) as stated in Section IV. In Fig. 6 we show results for three different ratios of the quark number susceptibilities, $\left(\chi_{2}^{q} / T^{2}\right) /\left(n_{q} / T^{3}\right),\left(\chi_{3}^{q} / T\right) /\left(\chi_{2}^{q} / T^{2}\right)$ and $\left(\chi_{4}^{q}\right) /\left(\chi_{2}^{q} / T^{2}\right)$ (top to bottom). In addition to marking the Stefan-Boltzmann results (arrows on the right hand sides) we also show the results from the hadron resonance gas discussed in Section IV (dashed black lines in the confined region).

In the high temperature region all ratios show very stable plateaus with small errors. For all values of $\mu \beta$ the positions of the plateaus are in almost perfect agreement with the corresponding Stefan-Boltzmann results (the ratio $\left(\chi_{4}^{q}\right) /\left(\chi_{2}^{q} / T^{2}\right)$ is independent of $\left.\mu \beta\right)$. Below the crossover the error bars are much larger, and only the ratio $\left(\chi_{2}^{q} / T^{2}\right) /\left(n_{q} / T^{3}\right)$ can be studied in a quantitative way. However, for this case we find very good agreement with the model data, this time from the HRG.

Concerning $\left(\chi_{3}^{q} / T\right) /\left(\chi_{2}^{q} / T^{2}\right)$ and $\left(\chi_{4}^{q}\right) /\left(\chi_{2}^{q} / T^{2}\right)$, at least for the $8^{3} \times 4$ data where we have larger statistics, we find qualitative agreement with the HRG results, in particular the correct trend when increasing $\mu \beta$.

The overall assessment is that the ratios are very accurately described by the Boltzmann limit above the crossover and at least for the lowest ratio we could establish good agreement with the HRG. Between the two phases is a narrow region of transitory behavior connecting the two plateaus.

We conclude the presentation of our numerical results with an analysis of the critical line in the $\mu \beta-T$ plane. For that purpose we determined $T_{c}$ from the inflection point of our results for $\chi_{2}^{q} / T^{2}$ (compare the bottom plots in Fig. 4). In Fig. 7 we show the corresponding data points in the $\mu \beta-T$ plane using results from our $8^{3} \times 4$ and $12^{3} \times 6$ lattices. The two data sets have a small discrepancy which, however, never exceeds $3 \mathrm{MeV}$. This is a surprisingly good agreement, since for the $8^{3} \times 4$ one still expects sizable finite volume and discretization effects.

\section{SUMMARY}

In this article we present results for generalized quark number susceptibilities and their ratios at finite temper-

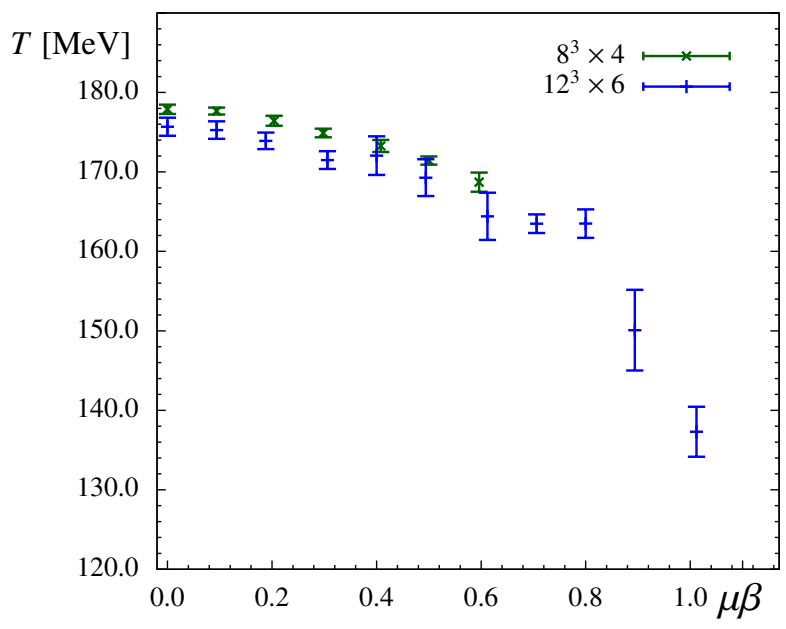

Figure 7: The critical line in the $\mu \beta-T$ plane, as determined from the inflection points of $\chi_{2}^{q} / T^{2}$.

ature and chemical potential. The calculation is based on the fugacity expansion which we show to be particularly well suited for quark number related observables. In particular we demonstrate that the susceptibilities can be obtained from moments of the expansion coefficients of the fugacity series, the so-called canonical determinants. The canonical determinants are needed very accurately for rather large values of the net quark number and we obtain them using Fourier transform with respect to imaginary chemical potential combined with a dimensional reduction technique. This numerical calculation is rather challenging, and part of the motivation for this article is to demonstrate, that the fugacity approach can indeed be extended to physically relevant volumes.

Our calculations are done for two flavors of Wilson fermions on two lattice sizes with different resolution and temperature values on both sides of the crossover. We study the generalized susceptibilities up to fourth order and on the finer lattice could extract reliable results up to $\mu \beta \sim 1.0$ with moderate numerical effort. The results for the susceptibilities agree with other lattice studies and for the ratios we reproduce the expected good agreement with the HRG below the crossover and with the StefanBoltzmann results above.

Although our results from the fugacity expansion are in good agreement with other calculations and the results from models, we are aware that the results presented here are still of exploratory nature. In particular a calculation on larger and finer lattices is needed which in turn would also allow one to use smaller quark masses. Increasing the volume will drive up the cost since the range of the net quark numbers for which the canonical determinants is needed is determined by the quark number susceptibility which is an extensive quantity. When lowering the quark mass studies in the free case suggest only a very weak increase in cost.

To conclude, we believe that the fugacity expansion has 
an interesting potential as a complimentary expansion to the Taylor series, with different properties (infinite Taylor series versus finite Laurent series), and different numerical challenges.

Acknowledgements: The authors would like to thank Andrei Alexandru, Szabolcs Borsanyi, Julia Danzer, Christian Lang, Bernd-Jochen Schaefer and Kim Splittorff, and Jac Verbaarschot for interesting discussions. H.-P. Schadler is funded by the FWF DK W1203, "Hadrons in Vacuum, Nuclei and Stars". This work is partly supported by DFG TR55, "Hadron Properties from Lattice $Q C D$ " and by the Austrian Science Fund FWF Grant. Nr. I 1452-N27.

\section{Appendix: Dimensional reduction}

In this paper we use a modification of the formula for the dimensional reduction derived in [9]. The modified form is more suitable for the applications discussed in this paper. In this appendix we use the conventions and the notation of [9]. Following [9], after dividing the lattice into four domains which contain different timeslices, and integrating out the fermion degrees of freedom in all domains separately one obtains (this is Eq. (10) in [9])

$$
\operatorname{det}[D(\mu)]=\tilde{A} H(\mu) .
$$

The factor $\tilde{A}$ is independent of the chemical potential and is given by a product of determinants of matrices

$$
\tilde{A}=\operatorname{det}\left[D^{(1)}\right] \operatorname{det}\left[D^{(3)}\right] \operatorname{det}\left[\tilde{D}^{(2)}\right] \operatorname{det}\left[\tilde{D}^{(4)}\right],
$$

where the $D^{(i)}$ denote the terms of the Dirac operator within the domains labeled by $i=1,2,3,4$ and the $\tilde{D}^{(i)}$ are the $D^{(i)}$ plus some additional terms (see Eq. (12) in [9] for further details). The $\mu$-dependent part can be written as

$$
H(\mu)=\operatorname{det}\left[1-H_{0}-e^{\mu \beta} H_{+}-e^{-\mu \beta} H_{-}\right],
$$

where $H_{0}, H_{+}$and $H_{-}$are three different, $\mu$-independent, $N_{s} \times 1 \times 3 \times 4$ dimensional matrices:

$$
\begin{aligned}
H_{0}= & \tilde{S}^{(4)} D_{1}^{(4,2)} \tilde{S}^{(2)} D_{1}^{(2,4)} \\
& \quad+\tilde{S}^{(4)} D_{3}^{(4,2)} \tilde{S}^{(2)} D_{3}^{(2,4)}, \\
H_{+}= & \tilde{S}^{(4)} D_{1}^{(4,2)} \tilde{S}^{(2)} D_{3}^{(2,4)} \\
H_{-}= & \tilde{S}^{(4)} D_{3}^{(4,2)} \tilde{S}^{(2)} D_{1}^{(2,4)}
\end{aligned}
$$

with $\tilde{S}^{(i)}=\left(\tilde{D}^{(i)}\right)^{-1}$, and the $D_{k}^{(i, j)}$ denote certain combinations of the Dirac terms connecting the domains and some $\tilde{S}^{(i)}$.

Now we want to rewrite Eq. (A.3) such that the matrices attached to the factors $e^{\mu \beta}$ and $e^{-\mu \beta}$ are hermitian conjugate to each other,

$$
\begin{aligned}
\operatorname{det}[D(\mu)] & \left.=\tilde{A} \operatorname{det}\left[1-H_{0}-e^{\mu \beta} H_{+}-e^{-\mu \beta} H_{-}\right]\right) \\
& \left.=\tilde{A} \operatorname{det}\left[1-\tilde{S}^{(4)} \tilde{H}_{0}-e^{\mu \beta} \tilde{S}^{(4)} \gamma_{5} H_{-}^{\dagger} \gamma_{5} \tilde{D}^{(4)}-e^{-\mu \beta} H_{-}\right)\right] \\
& =\tilde{A} \operatorname{det}\left[\tilde{S}^{(4)}\left(\tilde{D}^{(4)} \gamma_{5}-\tilde{H}_{0} \gamma_{5}-e^{\mu \beta} \gamma_{5} H_{-}^{\dagger} \gamma_{5} \tilde{D}^{(4)} \gamma_{5}-e^{-\mu \beta} \tilde{D}^{(4)} H_{-} \gamma_{5}\right) \gamma_{5}\right] \\
& =\tilde{A} \operatorname{det}\left[\tilde{S}^{(4)}\right] \operatorname{det}\left[\left(\tilde{D}^{(4)}-\tilde{H}_{0}\right) \gamma_{5}-e^{\mu \beta} \gamma_{5} H_{-}^{\dagger} \tilde{D}^{(4) \dagger}-e^{-\mu \beta} \tilde{D}^{(4)} H_{-} \gamma_{5}\right] \operatorname{det}\left[\gamma_{5}\right] .
\end{aligned}
$$

We have used the relations $\gamma_{5} \gamma_{5}=1$ and $\gamma_{5} \tilde{D}^{(4)} \gamma_{5}=$ $\tilde{D}^{(4) \dagger}$ and a relation that connects $H_{+}$and $H_{-}$(see [9]). In the last step we have grouped the terms in such a way that we can identify two new matrices

$$
\begin{aligned}
& K_{0}=\left(\tilde{D}^{(4)}-\tilde{H}_{0}\right) \gamma_{5}, \\
& K=\gamma_{5} H_{-}^{\dagger}\left(D^{(4)}\right)^{\dagger}=D_{1}^{(4,2)} \tilde{S}^{(2)} D_{3}^{(2,4)} \gamma_{5},
\end{aligned}
$$

where

$$
\tilde{H}_{0}=D_{1}^{(4,2)} \tilde{S}^{(2)} D_{1}^{(2,4)}+D_{3}^{(4,2)} \tilde{S}^{(2)} D_{3}^{(2,4)} .
$$

We end up with the modified formula for the dimensional reduction of the fermion determinant (compare Eqs. (9) and (10) in the main text),

$$
\operatorname{det}[D(\mu)]=A W(\mu \beta),
$$

where the $\mu$-dependent factor is now given by

$$
W(\mu \beta)=\operatorname{det}\left[K_{0}-e^{\mu \beta} K-e^{-\mu \beta} K^{\dagger}\right] .
$$

The factor $A=\tilde{A} \operatorname{det}\left[\tilde{S}^{(4)}\right]$ is still independent of the chemical potential and drops out in calculations of observables (see Section II). In contrast to three matrices in the original form of the winding expansion stated in [9], we now have just two distinct matrices $K_{0}$ and $K$. These matrices have to be pre-computed only once in the simulation code and can then be used in the calculation for different values of chemical potential as described in the main text of the paper. 
[1] D. Sexty, PoS LATTICE 2014 (2014) [arXiv:1410.8813]; C. Gattringer, PoS LATTICE $2013 \quad$ (2013) 002 [arXiv:1401.7788]; G. Aarts, PoS LATTICE 2012 (2012) 017, [arXiv:1302.3028 [hep-lat]]; L. Levkova, PoS LATTICE 2011 (2011) 011, [arXiv:1201.1516 [hep-lat]].

[2] J. Danzer, C. Gattringer, Phys. Rev. D 86 (2012) 014502, [arXiv:1204.1020 [hep-lat]].

[3] H.-P. Schadler and C. Gattringer, PoS LATTICE 2014 (2014), [arXiv:1409.4672 [hep-lat]].

[4] E. Grünwald, Y. Delgado Mercado, C. Gattringer, PoS LATTICE 2013 (2013) 448, [arXiv:1310.6520 [hep-lat]]. E. Grünwald, Y. Delgado Mercado and C. Gattringer, Int. Journal of Mod. Phys. A Vol. 29, No. 32 (2014), [arXiv:1403.2086 [hep-lat]].

[5] A. Alexandru, M. Faber, I. Horvath, K.-F. Liu, Phys. Rev. D 72 (2005) 114513, [arXiv:hep-lat/0507020].

[6] A. Hasenfratz and D. Toussaint, Nucl. Phys. B 371 (1992) 539.

[7] P. de Forcrand and S. Kratochvila, Nucl. Phys. Proc. Suppl. 153 (2006) 62 [hep-lat/0602024]; A. Li, A. Alexandru and K. F. Liu, Phys. Rev. D 84 (2011) 071503 [arXiv:1103.3045 [hep-ph]].

[8] B. Friman, F. Karsch, K. Redlich, V. Skokov, Eur. Phys. J. C 71 (2011) 1694, [arXiv:1103.3511 [hep-ph]]; A. Bazavov et al, Phys. Rev. D 86 (2012) 034509, [arXiv:1203.0784 [hep-lat]]; A. Bazavov et al, Phys.Rev. D88 (2013) 9, 094021, [arXiv:1309.2317 [hep-lat]]; S. Borsanyi, Z. Fodor, S. D. Katz, S. Krieg, C. Ratti, K. K. Szabo, Phys. Rev. Lett. 111 (2013) 062005,
[arXiv:1305.5161 [hep-lat]]; S. Borsanyi, Z. Fodor, S. D. Katz, S. Krieg, C. Ratti, K. K. Szabo, Phys. Rev. Lett. 113 (2014) 052301, [arXiv:1403.4576 [hep-lat]].

[9] J. Danzer, C. Gattringer, Phys. Rev. D 78 (2008) 114506, [arXiv:0809.2736 [hep-lat]].

[10] K. Nagata and A. Nakamura, Phys. Rev. D 82 (2010) 094027 [arXiv:1009.2149 [hep-lat]]; A. Alexandru and U. Wenger, Phys. Rev. D 83 (2011) 034502 [arXiv:1009.2197 [hep-lat]].

[11] MILC collaboration, http://physics.utah.edu/ ${ }^{\text {detar } / \text { milc.html }}$

[12] P. Braun-Munzinger, B. Friman, F. Karsch, K. Redlich, V. Skokov, Phys. Rev. C 84 (2011) 064911, [arXiv:1107.4267 [hep-ph]]. K. Morita, B Friman, K Redlich, V Skokov, Phys. Rev. C 88 (2013) 034903, [arXiv:1301.2873 [hep-ph]].

[13] A. Alexandru, C. Gattringer, H.-P. Schadler, K. Splittorff and J. J. M. Verbaarschot, arXiv:1411.4143 [hep-lat].

[14] R. Hagedorn, CERN Report 71-72 (1971). F. Karsch, K. Redlich, A. Tawfik, Eur. Phys. J. C 29 (2003) 549556, [arXiv:hep-ph/0303108]. S. Ejiri, F. Karsch, K. Redlich, Phys. Lett. B 633 (2006) 275-282, [arXiv:hep-ph/0509051]. A. Andronic, P. BraunMunziger, J. Stachel, M. Winn, Phys. Lett. B 718 (2012) 80-85, [arxiv:1201.0693 [nucl-th]].

[15] A. Nakamura and K. Nagata, arXiv:1305.0760 [hep-ph]. A. Nakamura and K. Nagata, Nucl. Phys. A 931 (2014) 825. 